OPEN

SUBJECT AREAS:

IMPLANTS

BIOMIMETICS

Received

11 December 2013

Accepted

17 April 2014

Published

13 May 2014

Correspondence and requests for materials should be addressed to H.R. (office@trauma. lbg.ac.at) or S.C.K. (kundu@hijli.iitkgp. ernet.in)

* These authors contributed equally to this work.

\section{Thromboelastometric and platelet responses to silk biomaterials}

\author{
Banani Kundu ${ }^{1 *}$, Christoph J. Schlimp ${ }^{2 *}$, Sylvia Nürnberger ${ }^{2}$, Heinz Redl ${ }^{2} \&$ S. C. Kundu'
}

'Department of Biotechnology, Indian Institute of Technology, Kharagpur-721302, India, ${ }^{2}$ Ludwig Boltzmann Institute for Experimental and Clinical Traumatology, AUVA Research Centre, Austrian Cluster for Tissue Regeneration, Vienna, Austria.

Silkworm's silk is natural biopolymer with unique properties including mechanical robustness, all aqueous base processing and ease in fabrication into different multifunctional templates. Additionally, the nonmulberry silks have cell adhesion promoting tri-peptide (RGD) sequences, which make it an immensely potential platform for regenerative medicine. The compatibility of nonmulberry silk with human blood is still elusive; thereby, restricts its further application as implants. The present study, therefore, evaluate the haematocompatibility of silk biomaterials in terms of platelet interaction after exposure to nonmulberry silk of Antheraea mylitta using thromboelastometry (ROTEM $\left.{ }^{\circledR}\right)$. The mulberry silk of Bombyx mori and clinically used Uni-Graft ${ }^{\circledR} \mathrm{W}$ biomaterial serve as references. Shortened clotting time, clot formation times as well as enhanced clot strength indicate the platelet mediated activation of blood coagulation cascade by tested biomaterials; which is comparable to controls.

T he fundamental of regenerative therapeutics exploits the self-healing ability of body by delivering cells, bioactive molecules/cues and providing mechanical or structural supports (if required) ${ }^{1}$. Biomaterials play significant role in regenerative therapy by acting as delivery vehicles, as cell scaffolding materials or implantable devices. The natural origin, wide range of cyto-compatibility, biodegradability, mechanical robustness and water based ease in processing into diverse morphologies including films, scaffolds, gels, micro and nano-particles encompass the application of silk protein fibroin in tissue engineering, biosensors and conformal devices ${ }^{2,3}$. The state of art of functional biomaterial designing requires compatibility with blood within the context of use as implantable medical device. Currently, there is no definite consensus about the haemocompatibility of silk biomaterials. Few studies are performed with mulberry silk solutions $s^{4}$, blended scaffolds ${ }^{5}$ and films ${ }^{6}$. All these studies assessed only one or few parameters of the blood coagulation cascade such as activated partial thromboplastin time $e^{7}$, prothrombin time $e^{8}$ and activation of platelets ${ }^{9,10}$ or formation of microparticle ${ }^{11}$ due to bloodbiomaterial interaction; without evaluating the whole blood. These approaches thus may end up with false negative or positive results (the limitations of the commonly employed techniques are summarized in detail in Supplementary Table 1). The recent attempt of using whole blood to investigate the haemocompatibility of silk biomaterials of mulberry silkworm Bombyx mori, evaluate the haemostasis based on platelet decay, formation of the thrombin-antithrombin complex and granulocyte-platelet conjugates in a 2D platform ${ }^{12}$. This study also corelates the crystalinity of silk biomaterials with haemocompatibility. However, for regenerative purpose, a 3D platform is favourable as it closely mimics the physiological condition. Blood-biomaterial interface plays key regulatory role, therefore, a more comprehensive real time understanding of 3D silk biomaterial mediated whole blood clotting process is needed for the fabrication of nonmulberry silk based vascular grafts.

Viscoelastic tests (VETs) such as thromboelastometry $\left(\right.$ ROTEM $\left.^{\circledR}\right)$ or thrombelastography $\left(\right.$ TEG $\left.^{\circledR}\right)$ are conducted as a point-of-care technique in clinical diagnostics ${ }^{13,14}$ as well as for in vivo and in vitro experimental research ${ }^{15-18}$. The employment of ROTEM/TEG provides the real-time kinetics of clotting initiation, clot formation, clot strength and clot breakdown, thus delivering a more complete picture of haemostasis. The test process is rapid, more functional assessment and considered to be superior over laboratory quantitative assessments. Furthermore, VETs are performed in whole blood including all cellular components that are usually removed by the centrifugation process when using plasma only. Therefore, VETs more closely recapitulate the in vivo coagulation system. TEG is already in use to evaluate the thrombogenicity (i.e. the ability of a material to induce the formation of clot or thrombus when coming in contact with blood) of insoluble biomaterials ${ }^{19}$. In line with these TEG experiments and for the purpose of the present study, the use of the NATEM-assay of ROTEM ${ }^{15,18}$, which is a re-calcified, non-activated test of citrated blood, is chosen to investigate the haemocompatibility of nonmulberry silk biomaterials. The practice of non-activated test is more rational and more appropriate, in contrast to other activated ROTEM assays such as the EXTEM assay (that extrinsically activates the coagulation) 

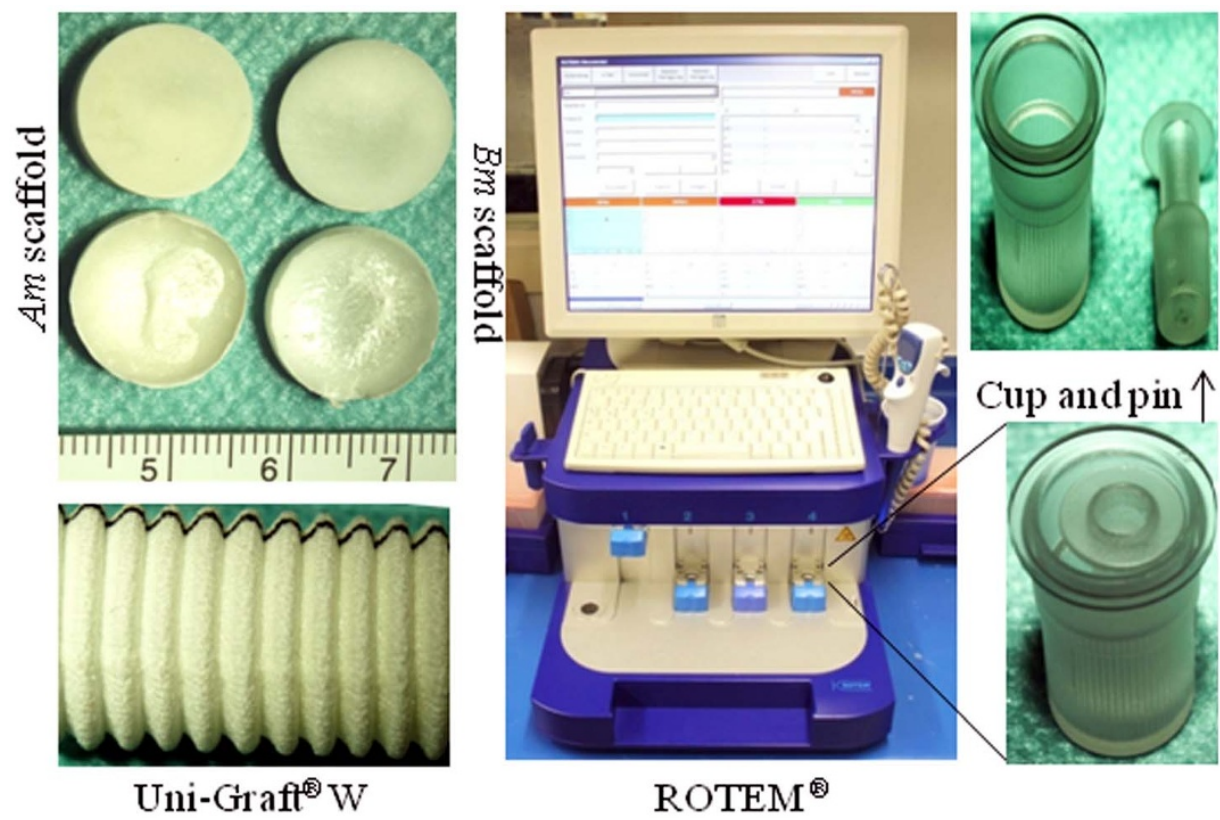

Figure $1 \mid$ Digital images of $A$. mylitta silk fibroin scaffold, $B$. mori silk fibroin scaffold, Uni-Graft ${ }^{\circledR}$ W and ROTEM ${ }^{\circledR}$. The measuring cell of ROTEM ${ }^{\circledR}$ consists of two parts; a pin and a cup. The pin acts as a sensor and rotating within the cup, which serves as cuvette.

and the INTEM assay (using intrinsic activation), which start coagulation already within 1-3 minutes, NATEM takes longer and is very sensitive to slow acting activators (biomaterials).

The aim of the current report is to evaluate the thrombogenicity of nonmulberry (Antheraea mylitta) silk protein fibroin 3D biomaterials along with silk fibroin scaffolds of mulberry (Bombyx. mori) and a clinical implant (Uni-Graft ${ }^{\circledR} \mathrm{W}$ ) as reference material using human whole blood, platelet-poor and platelet-rich plasma samples (the experimental outline is summarized in Supplementary Figure 1). Furthermore, to our best knowledge this is the first ever report of employment of thromboelastometry (ROTEM) for the in vitro haemocompatibility characterization of biomaterials. Additionally, the effect of agents that inhibit platelet function or aggregation is also observed in whole blood incubated with silk fibroin biomaterials in the same setting in order to provide a direction to manage the postimplant complication.

\section{Results}

Silk fibroin 3D scaffolds. The fabrication of silk fibroin scaffolds was started with aqueous silk fibroin solution of light milky white in colour in case of B. mori $(\mathrm{Bm})$ and transparent in case of A. mylitta (Am). During freezing, the water molecules of aqueous solution formed ice-crystals, which left void spaces after vacuum drying. The $3 \mathrm{D}$ porous constructs were then stabilized by the induction of $\beta$-sheet formation using ethanol. Each construct was opaque, cylindrical in shape with a diameter of $15 \mathrm{~mm}$, thickness of $2 \mathrm{~mm}$ and weighed $9 \mathrm{mg}$ (Figure 1). The pores of the silk scaffolds were heterogeneous in nature with average pore sizes of $113 \pm 21 \mu \mathrm{m}$ and $87 \pm 8 \mu \mathrm{m}$ for $A$. mylitta and $B$. mori scaffolds respectively (measured by Image J software [NIH version 1.46] from Supplementary Figure 2).

Blood collection. The volunteers ( 4 males and 2 females) donated blood for the study, were $30 \pm 6$ years old (range 20-40).

Whole blood thromboelastometry. Gathered stability data of the used experimental setting, i.e. Bm and Am versus control (Ctrl) at 0 , 60 , and $120 \mathrm{~min}$, tested in multiple NATEM series in whole blood samples from two volunteers, showed that both silk fibroin scaffolds, $\mathrm{Bm}$ as well as Am, exerted significant procoagulatory impact after 60 and $120 \mathrm{~min}$. This was observed in all four investigated ROTEM parameters and indicated by shortened CT (clotting time) and CFT (clot formation time); higher MCF (maximum clot firmness) and alpha angle value (Figure 2). Interestingly, between the volunteers, there was a significant difference in the onset of the procoagulatory influence of $\mathrm{Bm}$. In volunteer 1, compared to Ctrl, $\mathrm{Bm}$ did not reinforce blood coagulation after $60 \mathrm{~min}$, but after 120 min only.

After obtaining the stability data, a new experimental series with Ctrl blood and blood samples incubated with $\mathrm{Bm}$, Am as well as the vascular graft for reference was now performed in a larger group of blood samples from 5 different volunteers and with 4 measurements per group (Figure 3). All ROTEM parameters showed a procoagulatory pattern $(* \mathrm{p}<0.05)$ for all biomaterials (including the reference vascular graft). Furthermore, Bm and Am did reinforce blood coagulation as compared to the reference graft. Moreover, CT showed a faster onset in blood samples incubated with $\mathrm{Bm}$ as compared to Am $(* \mathrm{p}<0.05$, Figure 3$)$. CFT, alpha angle and MCF did not differ between the two silk fibroin scaffolds.

Plasma experiments with/without platelets. To exclude a possible influence of other blood cell components (i.e. red and white blood cells) another set of experiments was carried out in platelet-rich plasma (PRP). Measurements ( $\mathrm{n}=8$ per group) of native PRP (Ctrl) and PRP samples from one volunteer incubated with Bm, Am as well as the vascular graft for reference was now performed and showed a very similar pattern to whole blood. Reinforcement of ROTEM parameters was shown in Figure 4. When compared to control, the CT values were significantly reduced by $60 \%$ in $\mathrm{Bm}, 40 \%$ in Am and $20 \%$ in reference material (Figure 4$)(* \mathrm{p}<0.05)$. Furthermore, the CFT (and respective alpha angle values) corroborated with CT findings, but were not significantly different between Bm and Am. Interestingly, in PRP the incubation with biomaterials did not affect MCF and was also independent of scaffold types.

To prove that all previously seen procoagulatory effects of the biomaterials tested can be mainly attributed to platelets and was not reinforced by the native plasmatic coagulation components, we performed further measurements $(\mathrm{n}=4)$ in platelet-free plasma (PFP) and PRP of one volunteer for control (Figure 5). The results clearly demonstrated that in contrast to PRP, clotting time in PFP was extensively prolonged and not influenced by the silk-fibroin 

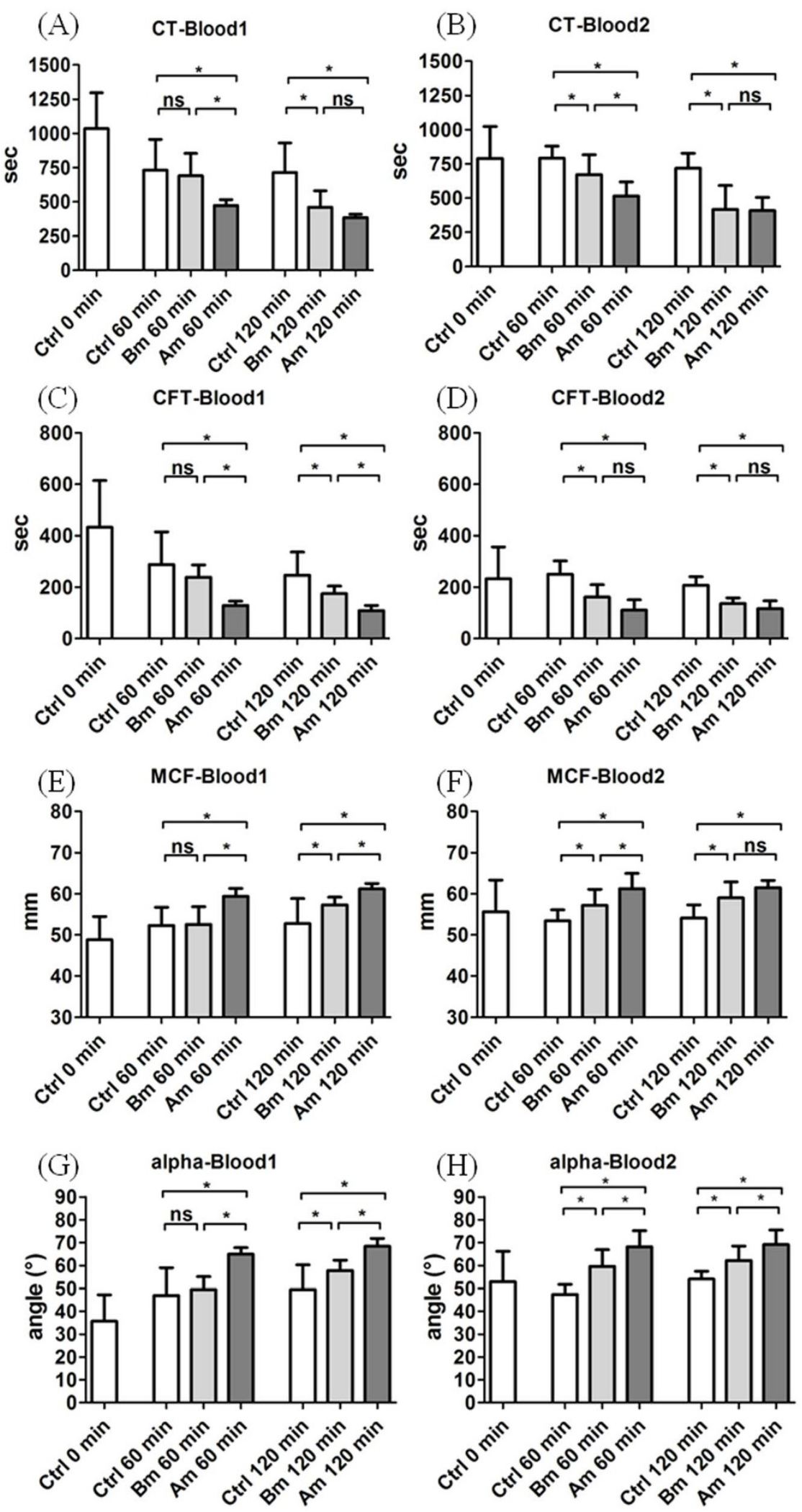

Figure $2 \mid$ Stability data of the experimental setting at 0,60 and 120 min in two volunteers with $n=63$ single measurements for Volunteer 1 and $n=71$ for Volunteer 2. (A-B) represent clotting time (CT); (C-D) represent clot formation time (CFT); (E-F) represent maximum clot firmness (MCF); (G-H) represent the alpha angle of clot formation. CT and CFT are significantly shortened, and MCF and alpha angle are significantly reinforced after 120 min in both Am and Bm as compared to Ctrl. Data shown are mean \pm SD. (ns $=$ not significant between the groups, ${ }^{*} \mathrm{p}<0.05$ between the groups).

scaffolds. It clearly indicated that the influence of experimental biomaterials on CT values was primarily a platelet-mediated effect.

Platelet quantity, morphology and state of activation were revealed in scanning electron micrographs (Figure 6). Scaffolds treated with platelet-poor plasma served as control. All the scaffolds exhibited porous surfaces. Scaffolds incubated with PRP exhibited platelets of dendritic shape with pseudopodia along with the completely stretched platelets throughout the surface irrespective of the scaffold 
CT reduction
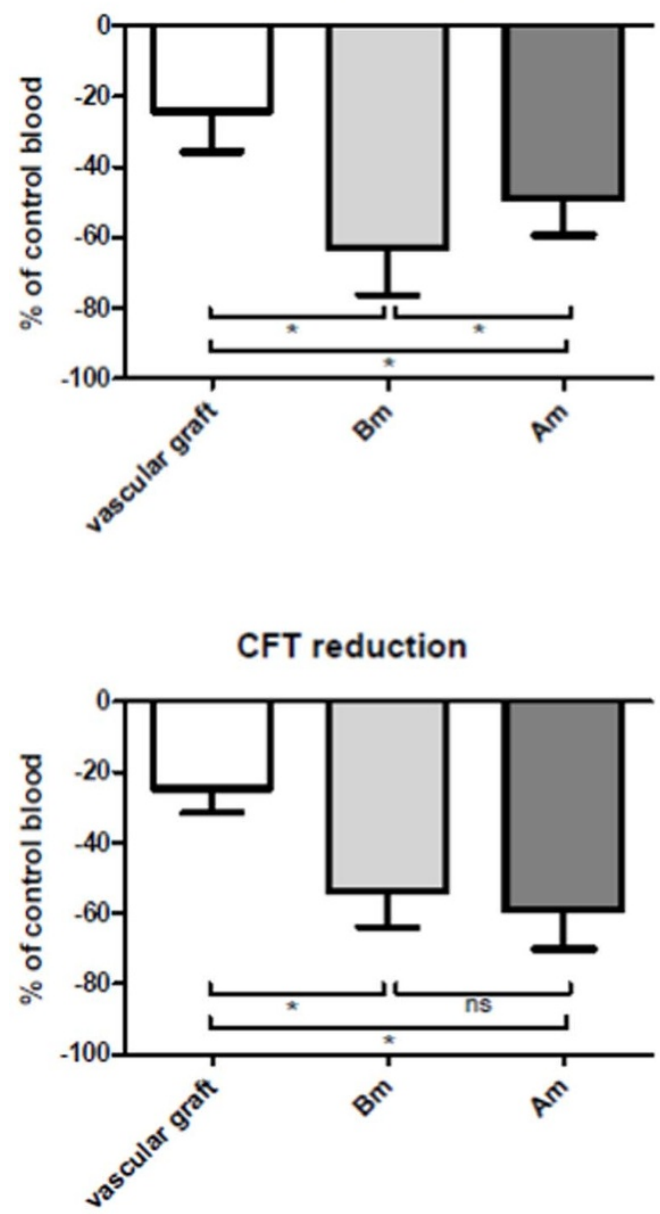

MCF increase
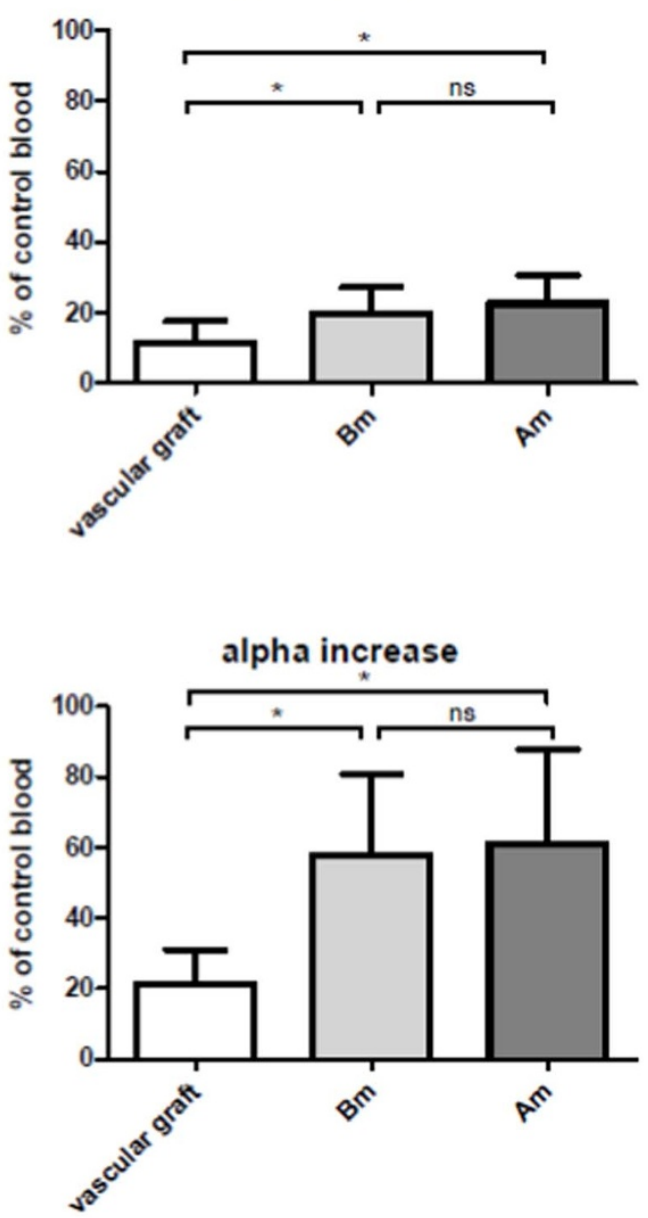

Figure 3 Four aliquots of freshly collected citrated blood from 5 volunteers was kept native for control or incubated with 3 biomaterials (vascular graft, $\mathrm{Bm}$ and $\mathrm{Am}$ ) over for a period of $2 \mathrm{hrs}$ and measured 4 times (each column represents a total of 20 measurements); Values are normalized as percentage of native control values at the same time point representing clotting time (CT), clot formation time (CFT), maximum clot firmness (MCF) and alpha angle of clot formation. Data shown are mean \pm SD. ${ }^{*}<0.05$ between the groups. All ROTEM parameters indicated initiation of procoagulant activity.

types. A few round non-activated platelets were observed on $\mathrm{Am}$ and reference material. Large aggregates of platelets prohibited the accurate individual count; therefore, the quantification of morphological changes was impossible ${ }^{20}$.

Response of anti-platelet therapeutics on whole blood coagulation. Since the key question of the present investigation was to evaluate the haemocompatibility of silk biomaterials, the influence of the clinically and experimentally used anti-platelet agents on whole blood, incubated with silk biomaterials, was also investigated. There was no significant inhibiting effect of aspirin, abciximab or cytochalasin D (Figure 7) on the procoagulatory behaviour (represented by clotting time) of blood incubated with silk biomaterials.

\section{Discussion}

In general, it is believed that contact activation of factor XII is the predominant activator of the intinsic coagulation cascade, when a biomaterial comes in contact with blood ${ }^{21}$. The major limitations of in vitro investigations are the absence of natural stimulus of complex haemostasis pathways and the cross-talk between primary and secondary haemostasis. Additionally, regular laboratory investigations employ plasma, which lack all blood cells and platelets. It is known that even erythrocytes may elicit coagulation via factor $\mathrm{IX}^{22}$.
Therefore, the current study involves the whole human blood to address the fundamentals of coagulation activation by silk protein fibroin biomaterials.

Processing parameters such as treatment temperature, solvents significantly influence the haemocompatibility of Bombyx mori silk fibroin films ${ }^{12}$. Freeze-drying method is thus used to fabricate $3 \mathrm{D}$ silk fibroin architectures for the present study. In freeze-drying method, the ice crystals act as porogens and circumvent the use of any inorganic chaotropic salts, which are marked unsafe in general practice ${ }^{23}$. Besides processing, the size and geometry of implants are also critical to evoke foreign body responses ${ }^{24}$. Therefore, it becomes necessary to assess the haemocompatibility of 3D silk fibroin scaffolds, since they are widely exploited as scaffolding materials for various tissues including bone, cartilage, nerve, cardiomyocyte and tendons ${ }^{3}$.

For fabrication of the vascular graft, the mechanical strength of the test biomaterial is also very important. The burst strength of the saphenous vein is $1,800 \mathrm{~mm} \mathrm{Ha}{ }^{25}$, which is considered as the gold standard. The cylindrical silk scaffolds $(10 \mathrm{~mm}$ diameter and $10 \mathrm{~mm}$ length) exhibit the mechanical strength of $136.26 \pm 41.09 \mathrm{kPa}$ and $50 \mathrm{kPa}$ for A. mylitta ${ }^{26}$ and $B$. mori $^{27}$ respectively. The reference biomaterial, Unigraft $\mathrm{W}$ is a well-established prosthetic graft ${ }^{28}$. It is woven polyster vascular prosthesis, which is impregnated with gelatin to improve its biocompatibility ${ }^{29}$. In contrast to Unigraft W, silk as a biocompatible material has thousand years of history (early 
CT reduction (in PRP)

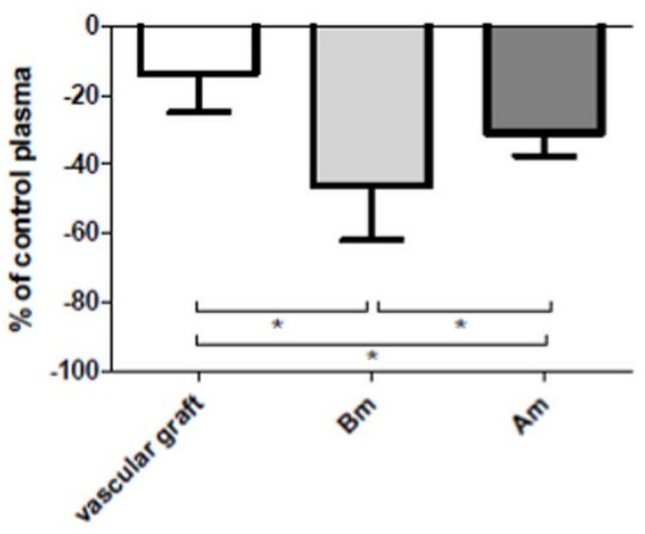

CFT reduction (in PRP)

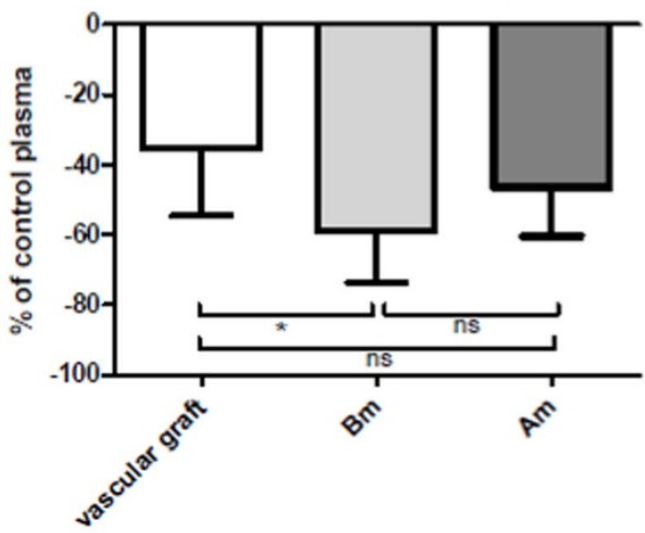

MCF increase (in PRP)

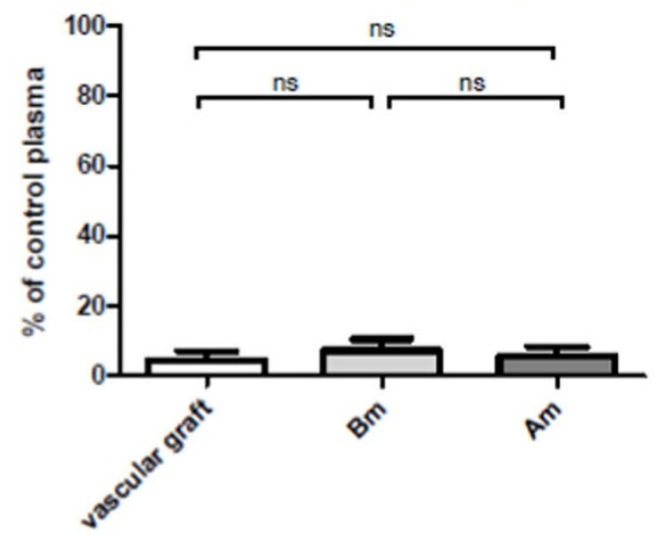

alpha increase (in PRP)

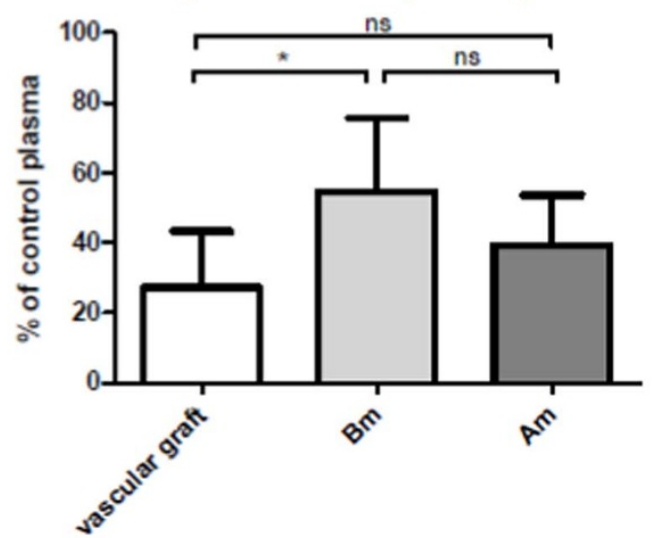

Figure 4 Biomaterials were incubated for $2 \mathrm{hrs}$ in platelet-rich plasma (PRP). Values are normalized as percentage of native control values at the same measurement time point: CT (clotting time), CFT (clot formation time), MCF (maximum clot firmness) and alpha angle. Samples from 2 volunteers with 4 measurements were involved in this experiment (each column represents a total of 8 measurements). Data shown are mean \pm SD. ${ }^{*} \mathrm{p}<0.05$ between the groups.

Egyptians used silk linen as suture $)^{30}$. Silk solution is also used as biocompatible coating materials ${ }^{31}$. The natural presence of integrin binding RGD sequences (tri-peptide sequence of Arg-Gly-Asp) in nonmulberry A. mylitta silk fibroin improves the proliferation of cells. The silk materials are natural in origin and available throughout the world; therefore, are relatively inexpensive. The processing of silk fibroins from their sources (cocoons or glands) is relatively easy and the resultant silk solution can readily be moulded into diverse morphologies ${ }^{3,32}$, which do not require any sophisticated instrument as compared to high tech-weaving technology employed in Unigraft fabrication. The amount of protein obtained from single gland or cocoon source is reasonably good to obtain enough amounts of $3 \mathrm{D}$ scaffolds used in present study. Hence silk fibroin has promising future as natural biomaterial in designing the artificial implants and other innovative devices ${ }^{33}$.

Viscoelastic tests (VETs), such as ROTEM or TEG are well established in clinical practice especially for trauma and the perioperative setting $^{13,14}$. VETs are also increasingly used for experimental research $^{15,16,18}$, but its implication in assessing the thrombogenicity of biomaterials is at its neo-natal stage ${ }^{19}$ However, the use of VETs in biomaterial science is gaining rapid popularity due to real-time comprehensive overview of clotting kinetics, minimal artefacts in coagulation activation, ease in sample preparation and relatively small sample volume ${ }^{19}$. The small sample volume $(300 \mu \mathrm{l})$ for one measurement allows using the blood of a single donor for a wide range of biomaterials, consequently eliminating the variation in blood sample from different donors and providing more reliable information of haemocompatibility of the biomaterials.

$\mathrm{CT}$ is proposed to be the most robust parameter in assessing the haemocompatibility or thrombogenicity of biomaterials via TEG ${ }^{19}$ and this is supportive to our present findings. However, we also found CFT, alpha angle and MCF are stable enough to significantly change with activation of coagulation cascade by biomaterials. When working with platelet inhibiting agents, CT might be the only parameter left for appropriate diagnosis, because remaining CFT, alpha angle and MCF may eventually depend on functional fibrinogen polymerisation only.

When comparing the altered ROTEM parameters between samples incubated with different scaffold materials, Bombyx mori (Bm) and Antheraea mylitta (Am) exert less haemocompatibility compare to the reference graft material, that itself also showed significant thrombogenicity as compared to native control (Figure 3). Platelet glycoprotein (GP) IIb/IIIa receptors have affinity for integrin binding sequences such as RGD (Arg-Gly-Asp) ${ }^{34}$. The presence of RGD sequence in nonmulberry silk fibroin of $\mathrm{Am}^{19}$ may interact with platelet membrane GP and imparts the difference in thrombogenicity than corresponding Bm biomaterials. MCF values of biomaterials may also corroborate with activated FXIII-platelet dependent thrombosis ${ }^{35,36}$. Additionally, the absence of any statistically significant difference of CT values between control and experimental groups in platelet-free plasma confirms the platelet-biomaterial interaction and platelet-mediated activation of coagulation cascade. 


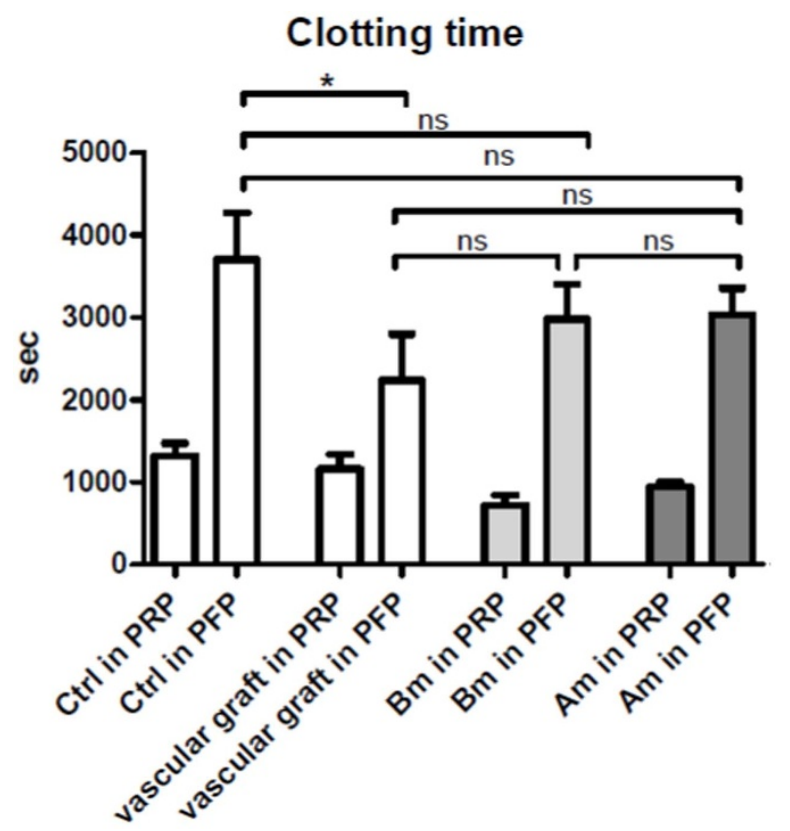

Figure 5 Biomaterials were incubated for 2 hrs in platelet-free plasma (PFP). CT (clotting time; absolute values in sec) of PRP (platelet-rich plasma) and PFP samples incubated with biomaterial. Data shown are mean \pm SD. ${ }^{*} \mathrm{p}<0.05$ between the groups.

Translating the observation of scanning electron microscopy, the completely spread pseudopodia and irregularly shaped platelets are indicative of platelet activation ${ }^{37}$. The morphological forms corresponding to activation degree of platelets are sequenced as round or discoid, early dendritic, spread dendritic, spread and fully spread ${ }^{38}$. All these forms are visible on the surface of biomaterials (Figure 6).

For future use of these biomaterials as implants, knowledge of suggestive immune-suppressors or anti-platelet therapeutics is the most coherent requirement of the current bio-medical practice. Antiplatelet agents, namely aspirin, abciximab and cytochalasin D are commonly applied to prevent the adverse events associated with platelet aggregation or for the laboratory use only (cytochalasin D). Aspirin interacts with intracellular signalling pathway and irreversibly inhibits the production of platelet cyclooxygenase, the key enzyme of synthesis of pro-aggregatory metabolites such as arachidonic acid, thromboxane $\mathrm{A}_{2}{ }^{34}$. Abciximab is a platelet glycoprotein (GP) IIb/IIIa receptor antagonist and cytochalasin D prevents the cytoskeleton reorganization in response to activation. Insignificant inhibition of the enhanced procoagulatory pattern of the tested biomaterial by aspirin, abciximab or cytochalasin $\mathrm{D}$ in the present study indicates that this pattern is not related to the mechanism inhibited by the commonly used agents. This may be due to the formation of microvesicles or exosomes by the activated platelets ${ }^{38}$. The exact physiological role of platelet-microvesicles, which are generated during wound bleeding or in certain clinical situation, is still elusive ${ }^{39}$. However, both procoagulant ${ }^{40}$ and anticoagulant ${ }^{41}$ activity of these vesicle is established. Additionally, these vesicle possess integrin binding sequences on their surfaces, which provides the answer to the relative difference of platelet activation by two different types of silk biomaterials in the present study.

The coating of the surface of silk biomaterials with anticoagulant agents may be one of the approaches to bypass/reduce platelet activation. Heparin, which is the most commonly used anticoagulant, prevents the thrombus formation or activation by interacting with antithrombin $\mathrm{III}^{42}$ and is commonly used anticoagulant coating. Further, the co-existence of heparin with antithrombin (covalent bonding) ${ }^{43}$ or chitosan (blending) ${ }^{44}$ result improved anti-coagulatory
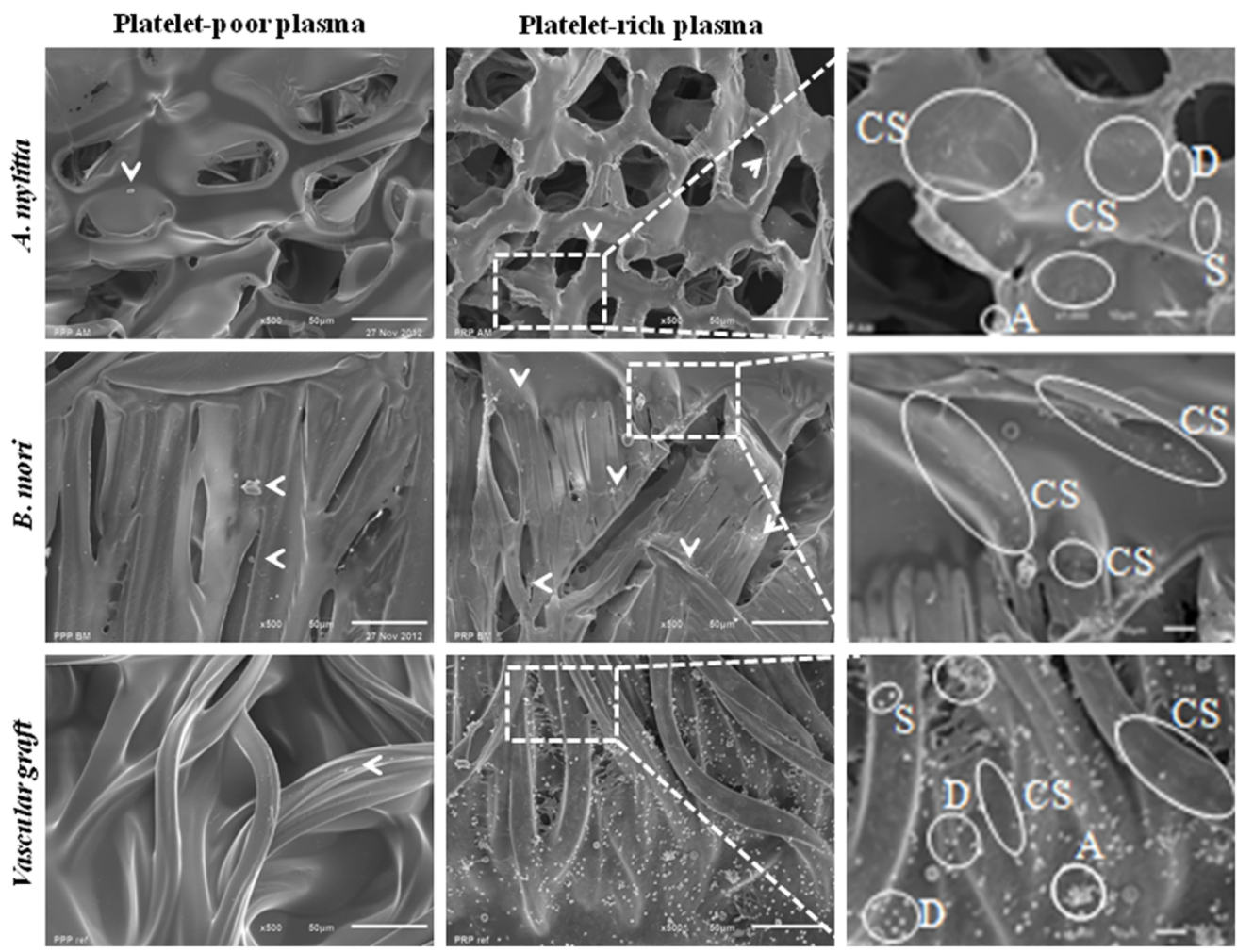

Figure 6 | Scanning electron micrographs of scaffolds incubated with platelet-poor plasma (PPP) and platelet rich plasma (PRP) for 2 hr with gentle end to end shaking after every 10 mins. Scale bar represented $50 \mu \mathrm{m}$ and $10 \mu \mathrm{m}$ respectively. Scaffolds incubated with PRP demonstrated the deformed activated platelets and platelet aggregates on surface. The various morphological forms were representative of different state of platelet activation: S-small; D-dendritic; A-aggregates; and CS-completely spread. Arrow heads indicate platelets. 
(a)

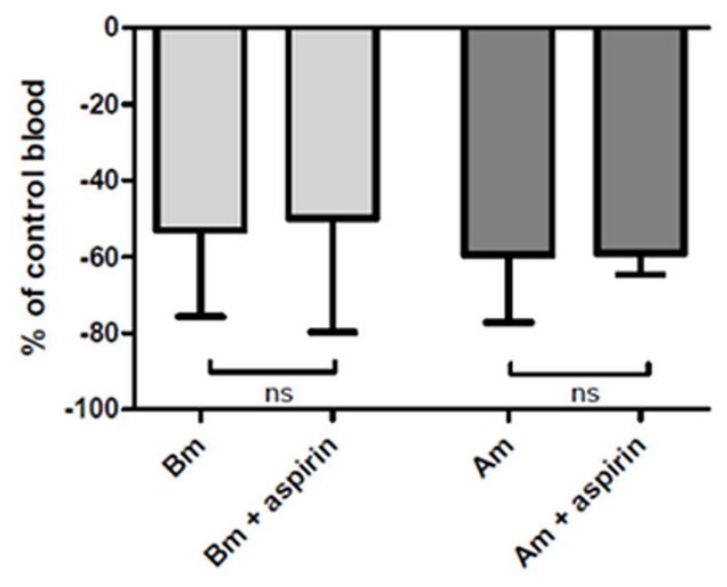

(b)

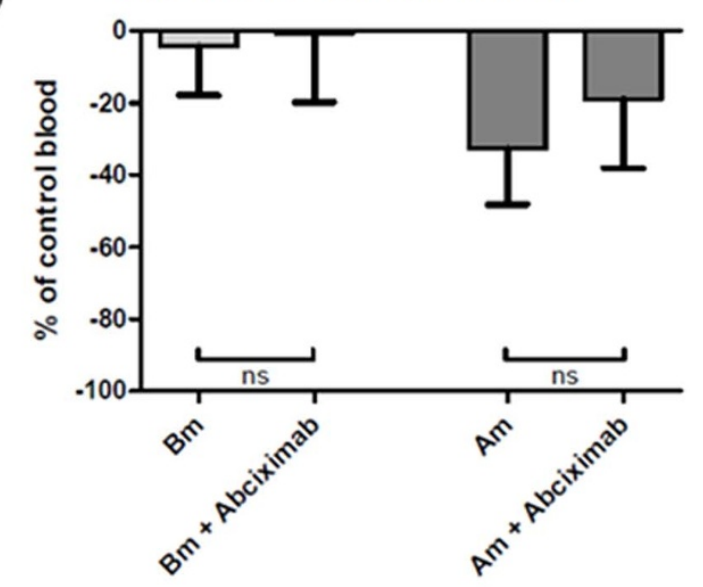

(c)

\section{CT reduction with CytD}

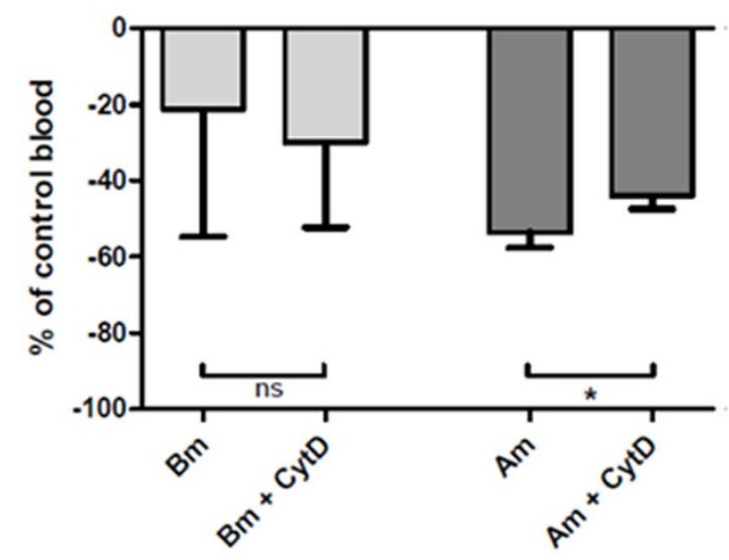

Figure $7 \mid$ Citrated blood of one volunteer was incubated with (a) aspirin $(0.1 \mathrm{mg} / \mathrm{ml}$ of blood), (b) abciximab (30 $\mu \mathrm{g} / \mathrm{ml} \mathrm{of} \mathrm{blood)} \mathrm{and} \mathrm{(c)} \mathrm{cytochalasin}$ $\mathrm{D}$ (CytD, $3.5 \mu \mathrm{g} / \mathrm{ml}$ of blood) for $10 \mathrm{mins}$ followed by exposure with silk biomaterials for a period of $2 \mathrm{hrs}$. The diagram showed clotting time (CT) values normalized to control blood at the same time point of measurement. Data were represented as mean \pm SD. ns $=$ not significant between the groups, each column represents a total of 4 measurements.

performance of biomaterials. Other attempts include the coating of methacryloylphosphorycholine laurylmethacrylate copolymer ${ }^{45}$, multi-layer of collagen and sulphated chitosan ${ }^{46}$, surface immobilization of phosphatidyl choline $e^{47}$ and ionical deposition of thermoplastic polyurethane on material surface ${ }^{48}$. The sulfonation of silk fibroin also improves its anticoagulant activity ${ }^{49}$.

\section{Methods}

Materials. After giving informed consent, six healthy adult human volunteers were included in the present study. It was ensured that the volunteers did not have any history of venous thromboembolism and did not use anti-inflammatory, anticoagulatory, anti-platelet or oral anti-conceptive medications. For every measurement series lasting 2 hours, blood was collected freshly and used immediately for the experimentation.

Mulberry silk cocoons of Bombyx mori (Bm) bivolatine silk cocoons were obtained from Bangalore, Karnataka State, India. The mature fifth instar silk larvae of Indigenous nonmulberry tropical tasar Antheraea mylitta (Am) were collected from local Tasar Sericulture Farm, West Midnapur of West Bengal State, India.

The commercially available Uni-Graft ${ }^{\circledR}$ W (B. Braun Melsungen AG, Berlin, Germany) single velour vascular prosthesis, which is clinically approved for the reconstructive cardiac and thoracic surgery, were used as reference material for the present study along with Bm silk scaffolds. This polyester prosthesis is impregnated with absorbable modified bovine gelatine to improve bio-absorbability and biocompatibility.

Preparation of silk protein fibroin solutions. Aqueous silk fibroin solution of $B$. mori and A. mylitta were obtained as described elsewhere ${ }^{50}$. Briefly, silk fibroin of $B$. mori was extracted from cocoons. The cut pieces of the cocoons were boiled in $0.02 \mathrm{M}$
$\mathrm{Na}_{2} \mathrm{CO}_{3}$ solution for $1 \mathrm{~h}$ to remove sericin. The degummed fibroin fibres were then rinsed thoroughly with distilled water and dissolved at $60^{\circ} \mathrm{C}$ in aqueous $9.3 \mathrm{M} \mathrm{LiBr}$ solution; followed by dialysis (MWCO 12000) against de-ionized water for $72 \mathrm{hrs}$. The silk fibroin of A. mylitta was collected from silk glands by dissecting the mature fifth instar larvae. Concisely, gland fibroin protein was squeezed into distilled water and dissolved in 1\% (wt\%) aqueous SDS solution containing $5 \mathrm{mM}$ EDTA and $10 \mathrm{mM}$ Tris ( $\mathrm{pH}$ 8.0). The silk solution was then dialyzed (MWCO 12000) against deionized water for $8 \mathrm{hrs}$. The final concentrations of both the silk fibroin solutions were adjusted to $2 \mathrm{wt} \%$ by using de-ionized water.

Fabrication of 3D silk fibroin scaffolds. The aqueous $2 \mathrm{wt} \%$ silk fibroin solutions were poured into moulds, frozen at $-20^{\circ} \mathrm{C}$ for overnight and lyophilized for $36 \mathrm{~h}$ to yield porous $3 \mathrm{D}$ silk fibroin scaffold $s^{50}$. The lyophilized scaffolds were briefly treated with absolute ethanol to induce crystallization and water insolubility in 3D porous network. The scaffolds were then rinsed thrice with $0.9 \% \mathrm{NaCl}$ solution before incubation with whole blood.

Blood collection and ROTEM analysis. ROTEM analysis of human volunteer blood and plasma samples ex vivo was approved by the local ethics committee (Ethikkommission für die Krankenanstalten der AUVA, Vienna, Austria), the methods were carried out in accordance with approved guidelines of the laboratory of the Ludwig Boltzmann Institute for Experimental and Clinical Traumatology, Vienna, Austria. After obtaining informed consent from all blood donors for this study, blood and plasma samples from six healthy adult human volunteers were included in the present study. It was ensured that the volunteers did not have any history of venous thromboembolism and did not use anti-inflammatory, anticoagulatory, anti-platelet or oral anti-conceptive medications. For every measurement series lasting 2 hours, blood was collected freshly and used immediately for the experimentation. 
Blood was drawn from the cubital vein through a 21-gauge needle using minimal stasis. The initial $2 \mathrm{~mL}$ of blood was discarded to avoid contamination of tissue thromboplastin due to needle puncture. Blood was then collected in $3.5 \mathrm{ml}$ tubes (Vacuette; Greiner Bio-One, Linz, Austria) containing $0.3 \mathrm{ml}$ buffered $3.2 \%$ trisodium citrate. Several aliquots of the blood samples were created for further testing.

Haemoglobin $[\mathrm{Hb}]$ concentration, blood cell counts (platelets [PLT], red blood cells [RBC], white blood cells [WBC] as well as their morphologic subgroups such as neutrophiles [NEU], lymphocytes [LYM], monocytes [MONO], eosinophiles [EOS] and basophiles [BASO]) were analyzed for each sample using an SF-3000 analyzer (Sysmex Corporation, Kobe, Japan) before proceeding with further experimentation. The respective normal reference values are: Hb 12.0-18.0 g/dL, PLT 130-400 K/ $\mu \mathrm{L}$, RBC 4.2-6.1 M/ $\mu \mathrm{L}$, WBC 4.8-10.8 K/ $\mu \mathrm{L}$, NEU 1.9-8, LYM 0.9-5.2, MONO 0.2-1.0, EOS $0.00-0.8$, BASO $0.00-0.2$. The normal volume of platelets is 7.2-11.1 fL.

To exclude a possible influence of other blood cells and to proof the impact of platelets only, we also conducted experiments in plasma with and without platelets. For this experiment, the collected blood was grouped into two categories: (1) one aliquot was used for platelet-rich plasma (PRP) and, (2) the second aliquot was used as platelet-free plasma (PFP). For PRP the blood aliquot was centrifuged at $115 \mathrm{~g}$ for $15 \mathrm{~min}$. To obtain PFP, the blood was firstly centrifuged at $2200 \mathrm{~g}$ for $15 \mathrm{~min}$, and then the clear supernatant (plasma) was centrifuged further at $2800 \mathrm{~g}$ for 15 mins followed by a frozen state at $-80^{\circ} \mathrm{C}$. The sample was then thawed at $37^{\circ} \mathrm{C}$ before experimentation and filtered through a $0.22 \mu \mathrm{m}$ membrane to remove also traces of residual platelets, resulting in $\mathrm{PFP}^{51}$.

Each scaffold was incubated with $2 \mathrm{~mL}$ of citrated blood/plasma for two hours at $37^{\circ} \mathrm{C}$ with a gentle end-to-end movement after every $10 \mathrm{~min}$. Uni-Graft ${ }^{\circledR} \mathrm{W}$ of equal weight of scaffold $(8.75 \pm 0.5 \mathrm{mg})$ was also incubated with citrated blood as reference material in the same way.

For baseline, a non-activated ROTEM analysis (NATEM) was carried out for all the investigations immediately after the collection of blood at $37^{\circ} \mathrm{C}$. In NATEM, the clotting cascade is activated by adding $20 \mu \mathrm{L}$ of star-tem ${ }^{\circledR}$ reagent $(200 \mathrm{mmol} / \mathrm{l}$ calcium chloride solution) to $300 \mu \mathrm{L}$ of citrated test blood with an automated and software guided pipette. The thromboelastometric changes were monitored in terms of clotting time (CT), clot formation time (CFT), alpha angle $(\alpha)$ and maximum clot firmness (MCF) at $30 \mathrm{~min}$ (=clot amplitude, A30) (the details of parameters included in ROTEM ${ }^{\circledR}$ study were summarized in Supplementary Table 2). ROTEM (TEM Innovation, Munich, Germany, and Fig. 1) uses a partially rotating pin that is vertically immersed into a pre-warmed cup containing the blood sample and can be performed simultaneously on four channels within the same device ${ }^{13}$ Coagulation of the (usually citrated) blood sample is initiated by re-calcification and is detectable because the formation of a fibrin clot between the pin and the cup wall reduces the rotation range of the pin. The generated signal is converted into a curve that indicates the time of clotting initiation, speed of clotting formation, clot strength and clot lysis.

Blocking of platelet activity. For experiments that intended to block platelet activity, $2 \mathrm{~mL}$ samples of whole blood, immediately after collection, were treated either with $200 \mu \mathrm{g}$ aspirin/acetylsalicylic acid (Aspisol ${ }^{\circledR}$, Bayer, Leverkusen, Germany), $60 \mu \mathrm{g}$ abciximab (ReoPro ${ }^{\circledR}$, Centocor B.V., Leiden, Netherlands), or $7 \mu \mathrm{g}$ cytochalasin D (Sigma Aldrich, Vienna, Austria) for 10 mins. These blood samples were then incubated with scaffolds under the same condition as described in section Blood collection and ROTEM analysis. Blood aliquots without any platelet antagonists were also incubated with respective scaffolds for each set and served as control

Scanning electron microscopy analysis. After incubation in platelet rich plasma and platelet free plasma for $2 \mathrm{hrs}$, scaffold samples were washed gently with phosphate buffer saline (PBS, pH 7.4) and fixed with $3.8 \%$ (v/v) paraformaldehyde solution. The scaffolds were dehydrated through a series of alcohol gradation, dried chemically with hexamethyldisilazane and sputter-coated with palladium gold. The scanning electron micrographs were obtained using a scanning electron microscope (SEM, JEOL JSM6510, Tokyo, Japan). The scaffolds incubated in PFP served as control for the study.

Statistical analysis. Data were represented as mean \pm standard deviation, a one-way repeated-measures analysis of variance (ANOVA) was used and a Newman-Keuls post-hoc correction for multiple comparisons was applied. When appropriate, the ROTEM parameters of samples incubated with the scaffolds were normalised to the respective controls in order to nullify the known variation in NATEM values ${ }^{18}$. Statistical analyses were carried out using GraphPad Prism 5 statistical software (GraphPad Software Inc., La Jolla, CA, USA). *P-values $<0.05$ were considered significant.

1. Williams, D. F. On the mechanisms of biocompatibility. Biomaterials 29, 2941-2953 (2008).

2. Omenetto, F. G. \& Kaplan, D. L. A new route for silk. Nat. Photonics 2, 641-643 (2008).

3. Kundu, S. C. et al. Non-mulberry Silk Biopolymers. Biopolymers 97, 455-467 (2012).

4. Lu, Q. et al. Cytocompatibility and blood compatibility of multifunctional fibroin/ collagen/heparin scaffolds. Biomaterials 28, 2306-2313 (2007).

5. Tamada, Y. Sulfation of silk fibroin by chlorosulfonic acid and the anticoagulant activity. Biomaterials 25, 377-383 (2004).
6. Motta, A., Migliaresi, C., Lloyd, A. W., Denyer, S. P. \& Santin, M. Serum protein absorption on silk fibroin fibers and films: surface opsonisation and binding strength. J. Bioact. Compat. Polym. 17, 23-35 (2002).

7. Lippi, G. \& Favaloro, E. J. Activated partial thromboplastin time: new tricks for an old dogma. Semin. Thromb. Hemost. 34, 604-611 (2008).

8. Murugesan, S., Park, T. J., Yang, H., Mousa, S. \& Linhardt, R. J. Blood compatible carbon nanotubes-nano-based neoproteoglycans. Langmuir 22, 3461-3463 (2006).

9. Snyder, T. A. et al. Preclinical biocompatibility assessment of the EVAHEART ventricular assist device: coating comparison and platelet activation. J. Biomed. Mater. Res. A 81, 85-92 (2007).

10. Basmadjian, D., Sefton, M. V. \& Baldwin, S. A. Coagulation on biomaterials in flowing blood: some theoretical considerations. Biomaterials 18, 1511-1522 (1997).

11. Gemmell, C. H., Ramirez, S. M., Yeo, E. L. \& Sefton, M. V. Platelet activation in whole blood by artifical surfaces: identification of platelet-derived microparticles and activated platelet binding to leukocytes as material-induced activation events. J. Lab Clin. Med. 125, 276-287 (1995).

12. Seib, F. P., Maitz, M. F., Hu, X., Carsten Werner, C. \& Kaplan, D. L. Impact of processing parameters on the haemocompatibility of Bombyx mori silk films. Biomaterials 33, 1017-1023 (2012).

13. Schöchl, H., Voelckel, W., Grassetto, A. \& Schlimp, C. J. Practical application of point-of-care coagulation testing to guide treatment decisions in trauma. J. Trauma Acute Care Surg. 74, 1587-1598 (2013).

14. Schöchl, H., Solomon, C. \& Voelckel, W. Thromboelastometry in the perioperative setting. Neth. J. Crit. Care 14, 23-31 (2010).

15. Schöchl, H. et al. Thromboelastometry (TEM) findings in disseminated intravascular coagulation in a pig model of endotoxinemia. Mol. Med. 17, 266-272 (2011).

16. Schöchl, H. et al. Similarities in Thromboelastometric $\left(\right.$ ROTEM $\left.^{\circledR}\right)$ Findings between Humans and Baboons. Thromb. Res. 130, e107-112 (2012).

17. Schlimp, C. J., Cadamuro, J., Solomon, C., Redl, H. \& Schöchl, H. The effect of fibrinogen concentrate and factor XIII on thromboelastometry in $33 \%$ diluted blood with albumin, gelatine, hydroxyethyl starch or saline in vitro. Blood Transfus. 13, 1-9 (2012).

18. Zipperle, J. et al. A novel coagulation assay incorporating adherent endothelial cells in thromboelastometry. Thromb. Haemost. 109, 869-877 (2013).

19. Shankarraman, V., Davis-Gorman, G., Copeland, J. G., Caplan, M. R. \& McDonagh, P. F. Standardized methods to quantify thrombogenicity of bloodcontacting materials via thromboelastography. J. Biomed. Mater. Res. B Appl. Biomater. 100, 230-238 (2012).

20. Hanke, A. A. et al. In Vitro impairment of whole blood coagulation and platelet function by hypertonic saline hydroxyethyl starch. Scand. J. Trauma Resusc. Emerg. Med. 19, 12-20 (2011).

21. Hong, J., Azens, A., Ekdahl, K. N., Granqvist, C. G. \& Nilsson, B. Material-specific thrombin generation following contact between metal surfaces and whole blood. Biomaterials 26, 1397-1403 (2005).

22. Iwata, H. et al. Purification, identification, and characterization of elastase on erythrocyte membrane as factor IX-activating enzyme. Biochem. Biophys. Res. Commun. 26, 65-70 (2004).

23. Stokols, S. \& Tuszynski, M. H. The fabrication and characterization of linearly oriented nerve guidance scaffolds for spinal cord injury. Biomaterials $\mathbf{2 5}$ 5839-5846 (2004).

24. Anderson, J. M. Inflammation, wound healing, and the foreign body response. [Ratner, R. D., Hoffman, A. S., Schoen, F. J. \& Lemons, J. E. (ed)] [165-172] (Academic Press, Boston, USA, 1996).

25. Nishibe, T., Kondo, Y., Muto, A. \& Dardik, A. Optimal prosthetic graft design for small diameter vascular grafts. Vascular 15, 356-360 (2007).

26. Mandal, B. B. \& Kundu, S. C. Non-mulberry silk gland fibroin protein 3-D scaffold for enhanced differentiation of human mesenchymal stem cells into osteocytes. Acta Biomater. 5, 2579-2590 (2009).

27. Nazarov, R., Jin, H. J. \& Kaplan, D. L. Porous 3-D scaffolds from regenerated silk fibroin. Biomacromolecules 5, 718-726 (2004).

28. Schmidtke, C. et al. First clinical results with the new sinus prosthesis used for valve-sparing aortic root replacement. Eur. J. Cardiothorac. Surg. 43, 585-590 (2013).

29. http://www.bbraun.com/cps/rde/xchg/bbraun-com/hs.xsl/products. html?prid= PRID00001953 [accessed on May 13th, 2014]

30. Leal-Egañna, A. \& Scheibel, T. Silk-based materials for biomedical applications. Biotechnol. Appl. Biochem. 55, 155-167 (2010).

31. Kundu, B. et al. Silk proteins for biomedical applications: bioengineering perspectives. Prog. Polym. Sci. 39, 251-267 (2014).

32. Rockwood, D. N. et al. Materials fabrication from Bombyx mori silk fibroin. Nat. Protoc. 6, 1612-1631 (2011).

33. Perrone, G. S. et al. The use of silk-based devices for fracture fixation. Nat. Commun. 5, 3385 (2014).

34. Ji, X. \& Hou, M. Novel agents for anti-platelet therapy. J. Hematol. Oncol. 4, 44-51 (2011).

35. Lauer, P. et al. Targeted inactivation of the mouse locus encoding coagulation factor XIII-A: hemostatic abnormalities in mutant mice and characterization of the coagulation deficit. Thromb. Haemost. 88, 967-974 (2002). 
36. Kasahara, K. et al. Impaired clot retraction in factor XIII A subunit-deficient mice. Blood 115, 1277-1279 (2009).

37. Goodman, S. L. Sheep, pig, and human platelet-material interactions with model cardiovascular biomaterials. J. Biomed. Mat. Res. 45, 240-250 (1999).

38. Heijnen, H. F., Schiel, A. E., Fijnheer, R., Geuze, H. J. \& Sixma, J. J. Activated platelets release two types of membrane vesicles: microvesicles by surface shedding and exosomes derived from exocytosis of multivesicular bodies and alpha-granules. Blood 94, 3791-3799 (1999).

39. Jy, W., Horstman, L. L., Arce, M. \& Ahn, Y. S. Clinical significance of platelet microparticles in autoimmune thrombocytopenias. J. Lab Clin. Med. 119, 334-345 (1992).

40. Zwaal, R. F. et al. Loss of membrane phospholipid asymmetry during activation of blood platelets and sickled red cells; mechanisms and physiological significance. Mol. Cell Biochem. 91, 23-31 (1989).

41. Tans, G. et al. Comparison of anticoagulant and procoagulant activities of stimulated platelets and platelet-derived microparticles. Blood 77, 2641-2648 (1991).

42. Capila, I. \& Linhardt, R. J. Heparin protein interactions. Angew. Chem. Inter. Ed. 41, 390-412 (2002).

43. Klement, P., Du, Y. J., Berry, L., Andrew, M. \& Chan, A. K. C. Blood-compatible biomaterials by surface coating with a novel antithrombin-heparin covalent complex. Biomaterials 23, 527-535 (2002).

44. Wei, H., Han, L., Ren, J. \& Jia, L. Anticoagulant surface coating using composite polysaccharides with embedded heparin-releasing mesoporous silica. Appl. Mater. Interfaces 5, 12571-12578 (2013).

45. Campbell, E. J. et al. Biocompatible surfaces using methacryloylphosphorylcholine laurylmethacrylate copolymer. ASAIO J. 40, M241-M915 (1994)

46. Li, Q.-L. et al. Anticoagulant surface modification of titanium via layer-by-layer assembly of collagen and sulfated chitosan multilayers. J. Biomed. Mater. Res. A 89A, 575-584 (2009)

47. Chandy, T., Das, G. S., Wilson, R. F. \& Rao, G. H. R. Use of plasma glow for surface-engineering biomolecules to enhance bloodcompatibility of Dacron and PTFE vascular prosthesis. Biomaterials 21, 699-712 (2000).

48. http://www.google.co.in/patents/US4678660 [accessed May 13th, 2014]

49. Liu, H., Li, X., Zhou, G., Fan, H. \& Fan, Y. Electrospun sulfated silk fibroin nanofibrous scaffolds for vascular tissue engineering. Biomaterials 32, 3784-3793 (2011)
50. Patra, C. et al. Silk protein fibroin from Antheraea mylitta for cardiac tissue engineering. Biomaterials 33, 2673-2680 (2012).

51. Schlimp, C. J. \& Zipperle, J. Viscoelastic reference values for maximum amplitude/ clot firmness of platelet-free plasma. Thromb. Haemost. 109, 356-357 (2013).

\section{Acknowledgments}

The work was financially supported by OEAD Indo-Austria collaborative fund, Department of Science and Technology, Department of Biotechnology (SCK), and Council of Scientific and Industrial Research (for individual fellowship to Banani), Govt of India, New Delhi. We thank Anna Khadem and Anton Klotz from the Ludwig Boltzmann Institute for Experimental and Clinical Traumatology for their technical assistance. One of the ROTEM devices used in this study was provided by TEM International.

\section{Author contributions}

B.K. and C.J.S. conceived, designed and performed the experiments. S.N. performed the sample preparation and scanning electron microscopy analysis. H.R. and S.C.K. supervised the laboratory analysis and data interpretation. B.K., C.J.S., S.N., H.R. and S.C.K. performed final manuscript preparation.

\section{Additional information}

Supplementary information accompanies this paper at http://www.nature.com/ scientificreports

Competing financial interests: The authors declare no competing financial interests.

How to cite this article: Kundu, B., Schlimp, C.J., Nürnberger, S., Redl, H. \& Kundu, S.C. Thromboelastometric and platelet responses to silk biomaterials. Sci. Rep. 4, 4945; DOI:10.1038/srep04945 (2014)

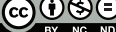

This work is licensed under a Creative Commons Attribution-NonCommercialNoDerivs 3.0 Unported License. The images in this article are included in the article's Creative Commons license, unless indicated otherwise in the image credit; if the image is not included under the Creative Commons license, users will need to obtain permission from the license holder in order to reproduce the image. To view a copy of this license, visit http://creativecommons.org/licenses/by-nc-nd/3.0/ 